\title{
De l'impossible coexistence ? De la nécessaire reconnaissance au fondement de la coexistence organisationnelle
}

Philippe Saielli et Isabelle Gillet-Roussel

\section{(2) OpenEdition \\ Journals}

Édition électronique

URL : http://journals.openedition.org/communicationorganisation/2896

DOI : 10.4000/communicationorganisation.2896

ISSN : $1775-3546$

Éditeur

Presses universitaires de Bordeaux

Édition imprimée

Date de publication : 1 mai 2004

ISSN : 1168-5549

Référence électronique

Philippe Saielli et Isabelle Gillet-Roussel, « De l'impossible coexistence ? De la nécessaire

reconnaissance au fondement de la coexistence organisationnelle », Communication et organisation

[En ligne], 24 | 2004, mis en ligne le 27 mars 2012, consulté le 19 avril 2019. URL : http://

journals.openedition.org/communicationorganisation/2896 ; DOI : 10.4000/

communicationorganisation.2896

Ce document a été généré automatiquement le 19 avril 2019.

(C) Presses universitaires de Bordeaux 


\title{
De l'impossible coexistence? De la nécessaire reconnaissance au fondement de la coexistence organisationnelle
}

\author{
Philippe Saielli et Isabelle Gillet-Roussel
}

$1 \mathrm{Lu}$ mode en gestion est généralement le reflet d'une pseudo réconciliation de l'économique et du social avec pour postulat une corrélation entre objectif de l'entreprise et objectif de l'individu. Or il s'agit là d'un cas particulier plutôt que la règle. Rares sont les situations où les objectifs personnels et organisationnels sont les mêmes. Très souvent le salarié doit tisser sa relation sur une trame d'entreprise rationnelle. La rationalité instrumentale ${ }^{1}$ qui se donne comme norme, parle du social. Comment pourrait-t-elle alors s'articuler avec l'expérience et le sens? De quelles manières contribue-t-elle à la violence symbolique, à la souffrance?

2 L'entreprise est une grande consommatrice de « concepts » : autonomie, projet, éthique. Chaque concept est souvent présenté comme le moyen de réconcilier l'économique et le social, de faire co-exister une logique instrumentale et une logique de sens. Chaque fois la communication implicite postule une coexistence possible: elle véhicule lu représentation d'un monde a-conflictuel, d'où sont absents les contradictions et les paradoxes. Pourtant en filigrane d'un questionnement concernant la coexistence des mondes organisationnels, il y a une problématisation du lien de l'homme dans sa dimension identitaire à l'entreprise. Cela s'inscrit dans une dynamique de débat qui est forcément tensionnelle et conflictuelle.

3 En prenant comme champ d'étude certains concepts clés du management, il s'agit de souligner la tendance de la rationalité instrumentale à instituer une communication qui s'autonomise de l'expérience des sujets pour instaurer une «emprise molle », désincarnée, qui tend à amalgamer sens et efficience. Il convient donc de réfléchir aux paradoxes induits par la recherche d'une coexistence et à la violence que génère une telle recherche. 


\section{La rationalité instrumentale : de l'efficacité à intersubjectivité ?}

4 L'organisation implique l'utilisation de la rationalité instrumentale pour mettre en mouvement les hétérogénéités. C'est la recherche du résultat dans une logique causeeffet qui organise l'entreprise, voir le modèle classique inspiré de Fayol et Taylor: Planifier-Organiser-contrôler-Performance, (avec retour de la performance sur la planification). C'est au management que l'on a confié la tâche de stabiliser les formes d'action cl de dépasser l'hétérogénéité des projets individuels et des antagonismes nés de l'action individuelle. Le processus de management existe dans toutes les organisations qui cherchent à atteindre un but afin de faciliter la coexistence des différentes ressources mobilisées pour atteindre les résultats escomptés.

Cette coexistence est en grande partie déterminée par le degré de centralisation de la structure mise en place et par la qualité du partenariat mis en œuvre.

Dans toute organisation, le dirigeant cherche à établir de manière explicite les liaisons et les rapports entre les individus et les groupes qui la composent. Les organigrammes, les descriptions de fonction, les procédures, la division du travail vont cadrer l'action collective et déterminer l'efficacité de cette action. Le management et la structure cherchent donc à maîtriser les hétérogénéités aliti d'assurer une coexistence performante. Il est important ici de souligner que pour une organisation, ce n'est pas la coexistence en soi qui est fondamentale, mais la coexistence en vue d'une recherche d'efficacité maximale.

7 D'emblée coexister dans les mondes organisationnels est problématique. Les organisations sont composées de parties hétérogènes qui ont chacune une logique propre. À litre d'exemple, on sait aujourd'hui que l'organisation représentée comme une culture dans laquelle tous les individus et groupes œuvrent pour un même objectif n'est qu'une représentation parmi tant d'autres. Cette représentation qui est portée par quelques acteurs, comme par exemple la direction des ressources humaines, entre en concurrence avec les autres représentations des différents services. À l'intérieur d'une même organisation les représentations concernant ce que sont l'organisation et la manière dont elle doit fonctionner sont multiples et souvent contradictoires ${ }^{2}{ }^{2}$ 'est la représentation inscrite dans les structures et les procédures qui va déterminer les comportements et les modes de relation des acteurs : c'est cette structuration qui conditionne les modalités de la coexistence.

Les formes du management et les structures ont certes évolué depuis Taylor. Chaque décennie apporte son lot de nouveautés. Les nouvelles formes d'organisation ont en commun de mettre en œuvre une "détaylorisation", c'est-à-dire de rechercher une coexistence organisationnelle qui laisse une plus grande part à l'autonomie des salariés, en leur permettant le contrôle des méthodes et des procédés d'exécution des tâches. Ces innovations managériales et structurelles sont caractérisées par la flexibilité, l'enrichissement des lâches, le développement des compétences, le management par projet. Le développement de la communication et des relations plus étroites entre les différents individus et groupes sont les pierres angulaires de tels dispositifs. Il s'agit donc de faire coexister harmonieusement l'économique et le social. 
9 Certes, il est essentiel de distinguer les discours des mises en pratiques réelles des entreprises. Mais au-delà de ce débat, on peut remarquer que pour faire coexister des individus et des groupes hétérogènes ayant leur propre logique d'action qui n'est pas nécessairement compatible avec la logique de l'ensemble, il y a une survalorisation de la pensée orientée vers la seule recherche de l'efficacité. Quelles que soient les formes que prennent les efforts pour faire coexister des logiques souvent antagonistes, elles témoignent toutes de la souveraineté accordée à la rationalité instrumentale.

En 1997 la revue internationale de psychologie consacrait un numéro à la question de «la résistible emprise de la rationalité instrumentale ». L'objectif de ce numéro était de décliner les significations de la rationalité instrumentale, de comprendre «comment et pourquoi tend-elle à prévaloir sur toute autre forme de pensée, affectant, de façon souvent inconsciente, les stratégies individuelles et collectives, les processus décisionnels, les relations sociales, et donc la gestion des entreprises, celles des institutions éducatives et de santé, les activités culturelles ou ludiques, la religion ou la recherche? $\|^{3}$.

11 La rationalité instrumentale est un mode de pensée logique qui permet la connaissance de l'objet qu'elle se donne, qui ne devrait ni être démentie par les faits occurrents ni entachée par la subjectivitét. L'essentiel est la maîtrise par la pensée de l'univers, des choses et des idées. Ce n'est pus le sens qui est visé, mais le savoir et la connaissance. Avec la rationalité instrumentale, il y a un déplacement de la vérité vers l'efficacité. Est vrai ce qui est efficace, c'est-à-dire une maîtrise rentabilisée ${ }^{5} \mathrm{Il}$ faut des solutions directement opérationnelles, préoccupation qui est accentuée par l'angoisse de perdre du temps. La rationalité instrumentale suppose une désimplication du sujet par rapport à son objet. Elle suppose l'acquisition d'une connaissance des faits en soi et d'une réalité des résultats non contestables parce qu'assurée ${ }^{6}$. Ce qui compte, c'est la nature du problème à résoudre et des solutions adéquates à ce problème. La seule préoccupation est l'atteinte et le dépassement des objectifs mesurables. Les intentions et les stratégies d'action sont appréhendées dans une perspective technicienne : elles sont ramenées à une logique de calcul. Tout est alors question de formalisation et d'outils. L'organisation est conçue comme un instrument au service de la rationalité instrumentale. La temporalité est celle $d u$ présent et l'avenir est une amplification du présent ${ }^{7}$. Cette apogée du recours aux fonctions de la pensée conduit au paradoxe suivant : il est impossible à la pensée de se penser elle-même. La pensée instrumentale se réfère à des interlocuteurs situés abstraitement (collaborateur, client, actionnaire, etc.). L'autre n'existe pas. il n'est que rouage de la logique cause-effet-solution : souvent il n'est qu'un rouage frustrant. Dans sa tendance à la maîtrise ${ }^{8}$, la rationalité instrumentale aboutit à la négation des autres formes de pensées et notamment des questions du sens.

La rationalité instrumentale est donc "tendance vers la maitrise ${ }^{9}$ ", " une aspiration à la toute puissance ». D'une part, il faut se délier de la subjectivité «la pensée rationnelle permet de décaper l'imaginaire et l'illusion, elle abolit l'indétermination et la subjectivitée ${ }^{10}$ ». D'autre part, la rationalité instrumentale, mathématique ou discursive, recourt à un mode de pensée logique qui permet la connaissance de l'objet qu'elle se donne, telle qu'elle ne devrait ni être démentie par les faits occurrents ni entachée par la subjectivité. Guist-Desprairies ${ }^{11}$ pose la question du recours à l'explicable - une rationalité causale, explicative, avide de solutions (mode du pragmatisme, du mode d'emploi, de la recette) - pour tenter d'avoir une représentation supportable de son identité et elle démontre le caractère bloquant et non régulateur du rationnel comme système défensif. Rendre compte d'une expérience n'est pas réductible à la description objectivante. La rationalité explicative « exclut une 
représentation du sens déterminé par des modalités particulières de relations à des objets ». " La recherche de sens n'est pas la recherche d'explications causales ni celle d'un sens caché à découvrir mais un processus de construction progressif, ouvert ${ }^{12}$ ». La rationalité instrumentale est une pensée bloquante qui vise le contrôle, qui devient à l'extrême une énergie de maîtrise. La rationalité instrumentale peut alors devenir un mécanisme de défense subtile permettant de lutter contre l'angoisse ${ }^{13}$.

La coexistence est ici pensée uniquement en termes d'efficacité à atteindre. En poussant à l'extrême cette logique, on peut considérer que la question de la coexistence ne se pose plus. En effet coexister se réfère au fait d'exister ensemble. Il serait d'ailleurs plus judicieux d'utiliser une scansion entre co et exister. Ce trait d'union "co-exister" permettrait de symboliser l'espace d'une différenciation. La problématique de la «coexistence" est bien une tension toujours en mouvement entre une dynamique d'intégration et une dynamique de différenciation, C'est dans cet équilibre toujours instable que le sujet peut se positionner, entre su fonction d'acteur et celle de sujet. Ce trait d'union rend compte d'une respiration, d'un espace de parole et du conflit possible dans lequel chacun peut se dire et surtout choisir son positionnement en acceptant la castration liée à son engagement.

co-existence renvoie à la notion d'interaction. Avec la coexistence, il ne s'agit plus de penser les individus indépendamment les uns des autres en fonction de leurs caractéristiques, mais comme produit de leur interaction. Le problème est qu'avec la rationalité instrumentale il y a un déni de l'interaction. La coexistence est pensée en termes de juxtaposition des individus et des groupes. À cet égard le recours à la notion de compétence est révélateur de ce phénomène. La tendance est d'inscrire la compétence dans le projet professionnel de l'individu, le rendant ainsi responsable de son évolution de carrière. On occulte le déterminisme des structures ainsi que le fait que la compétence n'a de sens qu'en interaction avec une situation de-travail et en lien avec les autres personnes.

donc que la coexistence lorsqu'elle s'inscrit dans la rationalité instrumentale devient un moyen de contrôle des comportements au travail. Coexister est alors défini par une modélisation mécaniste limitant l'initiative et l'originalité de la personne à ses tâches. Cette représentation instrumentale occulte la personne en tant que producteur de sens, capable de stratégie et d'innovation.

Toutes les formes du management moderne, notamment le «management de l'excellence " n'ont jamais réussi à penser la coexistence autrement que de manière instrumentale. Certes, on a attribué aux individus juste assez de pouvoir pour devenir responsables sans pour autant qu'on leur accorde le moindre droit quant aux choix et à la détermination politique de ceux-ci. Le management de l'excellence donne l'illusion aux individus qu'ils sont autonomes, en fait ce sont la structure et les procédures de gestion qui déterminent les objectifs. Parler d'impossible coexistence, c'est mettre en évidence qu'une conception mécaniste du fait d'exister ensemble rend caduque toute forme d'acceptation de l'intersubjectivité. Il ne peut y avoir profondément coexistence que lorsqu'il y a au préalable acceptation du deuil de la toute-puissance et acceptation du manque. C'est cette rupture qui ouvre au fait que les protagonistes d'une situation sociale peuvent être pensés comme « coauteurs altérant la matière de leurs échanges à travers des jeux de réappropriai ion, des commentaires, des interprétations $»^{14}$

Il s'agit alors de concevoir la co-existenee non pas à partir d'une causalité linéaire, mais en fonction de formes maillées fondées sur le jeu interactif des significations. D’emblée la 
co-existence suppose une lecture plus complexe de l'organisation. Même si l'inconscient, l'habitude, l'idéologie, le consensus social, se liguent pour imposer des réalités évidentes, qui masquent des phénomènes plus complexes que ce que la raison ou la perception réaliste veut faire croire.

\section{Une autonomisation paradoxale de la rationalité instrumentale.}

18 Le paradoxe est que la rationalité instrumentale "s'autonomise» pour devenir une normalisation, s'invitant dans la définition des finalités et déniant toutes les autres logiques. Alors la rationalité instrumentale devient un instrument de normalisation et un mode idéal d'agir. Notamment, parce qu'on occulte le fait que la rationalité instrumentale n'est qu'un outil et devient le seul processus de pensée, un substitut de finalité d'où l'équation : rationalité instrumentale efficacité finalité.

On constate donc le phénomène suivant : à l'origine la rationalité instrumentale est un moyen efficace de mise en coexistence des différents individus et groupes de l'organisation. Puis cette rationalité s'est imposée, coexister se résume alors à une maîtrise rentabilisée. Elle sert à créer une illusion de maîtrise qui conduit à traiter la coexistence comme un objet extérieur aux personnes, sur lequel il est possible d'intervenir de manière technique en excluant les affects et les questions du sens de l'action. Ainsi, la rationalité instrumentale « s'autonomise " s'invitant dans la définition des finalités et déniant toutes les autres logiques. Ce phénomène est explicite dans les cas de restructuration des entreprises. Comme le montrent Duboulov et labre ${ }^{15}$, il y a un refus de penser la souffrance dans les entreprises qui se traduit par un envahissement de l'émotionnel, des passages à l'acte ou au contraire une hyper rationalisation. Le refus de la réalité (de la perte en général et de la souffrance en particulier) peut prendre la forme paradoxale d'hyper-adaptation au discours de la logique économique de l'entreprise, la rationalisation de la situation, C'est l'acceptation d'une fatalité ${ }^{16}$, la soumission au discours ambiant : il n'y a pas moyen de faire autrement, C'est lu «foi du Marché », «la Volonté des Actionnaires » auxquels il faut se soumettre et qui s'impose à tous. C'est bien connu que se livre une guerre sans merci dont les licenciés et autres laissés pour compte sont les victimes «naturelles». Il y a un enfermement collectif dans cette logique qui neutralise toute autre perspective.

\section{De l'instrumentalisation des concepts}

Il est également possible de rendre compte de celte autonomisation de la rationalité instrumentale en étudiant l'évolution de concepts qui ont été présentés comme moyens d'améliorer la coexistence entre l'économique et le social : autonomie, projet, éthique. L'usage de ces trois derniers mots aux valeurs positives se présente comme un horizon fantasmatique de maitrise, d'homogénéité aussi puisque ces mots traversent toute la société, en sont « l'air du temps».

Un concept suppose une zone de validité limitée, un champ disciplinaire, au risque de diluer son acception dans le sens commun, et bien au-delà d'en diluer les finalités. Or autonomie, concept surinvesti, et projet appartiennent à de multiples champs, et leur inscription dans le discours entrepreneurial est en rupture forte avec d'autres champs: 
l'autonomie en pédagogie est une question d'émancipation au service du sens du projet de vie de l'apprenant et non un levier d'efficacité. L'autonomie en organisation est encadrée : «le salarié sera demain plus autonome mais plus contraint et soumis au système ${ }^{17}$ ». En outre l'aura du concept de " projets " peut être le fait d'une « ligure idéalisée »" ${ }^{18}$.

L'autonomie en entreprise est mise en relation avec l'efficience et non avec le sujet. Avec la rationalité instrumentale, il y a un déplacement de la vérité vers l'efficacité. Est vrai ce qui est efficace, c'est-à-dire une maîtrise rentabilisée. Le critère recherché et récurrent énoncé lors d'une enquête auprès de tuteurs de stagiaires en alternance ${ }^{19}$, est bien l'autonomie au sens de l'économicité du temps plus que de la maturation du stagiaire. Le processus d'internalisation et de rationalisation touche aussi l'autonomie comme l'écrit Ibanez : «nous ajustons nos croyances à ce que nous faisons réellement pour pouvoir continuer à penser que nous sommes des êtres doués d'autonomie, c'est-à-dire pour pouvoir continuer ci penser que ce que nous faisons correspond à ce que nous croyons ${ }^{20}$. Ce qui interroge les liens entre la pratique et l'idéologie. Citons aussi les multiples éludes sur la déviance de salariés intégrée et encouragée par les entreprises, soulignant la «vraie fausse autonomie accordée ». "L'autonomie de la personne ne tient pas seulement à la dévolution, au contrôle et au partage du pouvoir, mais encore à l'autorisation, capacité de se faire soi-même auteur, renouvelant le vécu de I autoritée ${ }^{21} »$.

23 Un projet est une action mobilisant des ressources, limitée dans le temps et orientée vers un but. La cité par projet, ainsi nommée par le sociologue Boltanski ${ }^{22}$, comme nouvel appareil justificatif, est rendue historiquement explicable par l'explosion des NTIC, du réseau, de l'attention à la flexibilité, au changement, à l'incertitude ("l'épreuve pour l'homme connexionniste sera le passage d'un projet à l'autre "23). de l'émergence du management moins hiérarchique, héritage post 68 et donc d'une recherche de structures peu hiérarchisées et d'une volonté d'optimisation des compétences. La valorisation des propriétés relationnelles dans ce mode de coordination transversale (non synonymes des propriétés propres aux sujets) est le fruit des travaux de communication, de psychologie sociale et d'anthropologie sociale. Le projet est décrit comme "un amas de connexions actives ». "une poche d'accumulation temporaire " ${ }^{24}$. L'entreprise ne peut être à court de projets et elle tisse une constellation. Voici l'interdépendance consacrée, le temps de la trajectoire pris dans un espace du mai liage. De fait la clôture, le passage d'un projet à l'autre sont institutionnalisés, consécutifs au compromis entre autonomie et dimension sécuritaire. Il n'y pas de temps pour accepter le deuil engendré par la fin d'un projet. On va faire des bilans, des synthèses pourannuler l'impact affectif de lu tin. Tout est rationalise ${ }^{1} \mathrm{y}$ compris et surtout les manifestations affectives.

La flexibilité et l'adaptabilité sont ici des qualités qui ne relèvent pas de la docilité. Le "grand", ainsi nommé par Boltanski, dans un monde connexionniste est actif et autonome. Il est "un - adar $»^{25}$ choisissant avec discernement ses réseaux, ses relations, évitant de se connecter à des personnes qui « occupant des positions proches, risquent de ne lui apporter que des informations et des liens redondants " $^{26}$. "L'ipséité qu'il se reconnaît n'est pas le résultat d'une dotation préexistante, ni même celui d'une trajectoire ou d'une expérience. Elle dérive de la constellation des connexions établies. Chacun n est lui-même que parce qu'il est les liens qui le constituent $»^{27}$. La raison instrumentale se dote d'un autrui instrumentalisé ; L'intersubjectivité est mécanisée. La tension est intégrée entre permanence du soi et adaptabilité dans des liens transitoires, engagement/désengagement. 
25 La mise est payante : autonomie (encadrée) de l'individu respectée, projet restituant la relation comme essentielle (mais au sens du réseau activé et utile), éthique sécurisante (qui supprime le débat).

Selon Bergmann ${ }^{28}$. "respecter une certaine éthique en affaires revient à attribuer des valeurs aux diverses conséquences des activités économiques et à établir des priorités entre elles. Ainsi comprise, l'éthique en gestion ne consiste pas en un canon mais en un processus: celui d'une réflexion continue sur les conséquences multiples des actions ». La question de l'éthique ${ }^{29}$ met en évidence la pluralité des valeurs qui coexistent dans les organisations. Parler d'éthique c'est alors admettre que la logique économique n'est pas la seule manière de discriminer ce qu'il est bien ou mal de faire dans une organisation. C'est ouvrir le débat, c'est introduire des questionnements liés aux conflits et aux ajustements de valeurs dans les pratiques de management. Plus précisément, on parle de délibération pour désigner la volonté d'ordonnancer et d'ajuster les multiples valeurs qui sous-tendent l'agir et les conséquences proches ou lointaines du choix de l'action. Elle se présente comme une instance dynamique, un processus rationnel, permettant aux managers de prendre en compte la dimension éthique de leur pratique. Ce qui va à l'encontre d'une représentation homogène de l'organisation et notamment une homogénéité des valeurs au sein de l'entreprise: par exemple, l'idée selon laquelle en travaillant pour les objectifs de l'entreprise, l'individu travaille à son propre développement. La délibération est en l'ait légitimée par deux éléments qui s'opposent à cette homogénéisation: la liberté de l'acteur et la multitude des référentiels axiologiques au sein de l'entreprise.

Lorsqu'on examine l'évolution des discours et des pratiques en manière d'éthique d'entreprise, on se rend compte que très souvent l'éthique devient un outil de gestion. L'éthique est alors confrontée au paradoxe suivant : en instaurant un système de valeurs pour encadrer et contrôler les comportements des personnels, l'éthique organisationnelle court le risque de devenir une morale close au sens de Bergson ${ }^{30}$. Si la définition d'obligation ne s'accompagne pas de la définition d'une forme de liberté qui garantisse à la fois la liberté de se contraindre et celle de le faire par sa propre volonté, alors l'éthique organisationnelle se ferme sur elle-même pour n'être plus qu'une éthique partiale et partielle. Ainsi le risque de l'éthique en tant qu'outil de gestion est de ne pas être un moyen en vue d'une fin plus globale-(le respect de l'environnement social par exemple) mais une fin en soi.

Pour devenir sujet de son action, la personne a besoin de s'approprier sa décision en lui donnant un sens et une direction, en fonction de valeurs auxquelles elle s'identifie, fin ne donnant pas cette liberté de sens aux acteurs et en ne créant pas d'espace pour penser l'agir par soi-même, on risque de voir naître des formes d'inhibitions. L'instrumentalisation de la question éthique conduit à une représentation qui se veut universelle et qui occulte la contingence des situations. La tendance est alors de s'accrocher à des modèles stéréotypés ou globalisants alors que les responsables ont de plus en plus besoin d'une connaissance fine des situations. Le risque est aussi de céder à la tentation de reproduire des schémas normatifs, sans tenir compte des logiques alternatives que les personnes développent pour donner un sens à leurs actes. En voulant faire de l'éthique un mode de régulation rationnel et objectif, on réduit la complexité des situations de gestion en enfermant le manager dans une représentation normative et universelle de la réalité. On ne l'aide ni à ouvrir ses compétences face à un problème de gestion, ni à prendre en compte la reconnaissance de l'autre. L'éthique devient alors répressive et entrave l'individu dans son évolution vers une autonomie personnelle à 
l'égard de l'action. Le risque est d'assimiler la représentation normative de la réalité au réel lui-même. On assiste alors à la naissance d'un «social gadgétisé » de courte durée dont l'essentiel est de créer de l'image ${ }^{31}$.

La difficulté de créer des espaces de mise en tension, en dialectique des logiques instrumentales et de sens génère une violence symbolique, un conflit non-dit. L'utilisation de concepts leurrants par l'entreprise participe du code de cette violence. Hors du discours articulé autour de concepts positifs, l'entreprise peut également instrumentaliser un concept perçu tout à fait négativement. La littérature sur le concept de harcèlement signale assez clairement qu'il peut être «l'expression" du pervers narcissique mais aussi l'outil silencieux de néomanagement par l'entreprise, une récupération rationnelle d'une violence psychologique.

À l'origine, le concept de harcèlement permet de rendre compte de la souffrance vécue par certaines personnes: il offre un sens et des mots à une douleur difficilement communicable. Très vite le concept tend à s'appliquer à toutes les situations. Tout devient alors harcèlement, indépendamment du vécu des personnes. Le harcèlement part donc d'un vécu, d'une souffrance individuelle. Mais le paradoxe, lié à l'idéologie dominante, est qu'on a privatisé le concept d'harcèlement, faisant passer au second plan l'inscription collective et conflictuelle $\mathrm{du}$ fondement $\mathrm{du}$ harcèlement. «La privatisation $d u$ conflit collectif en crise personnelle est l'une des grandes réussites de l'idéologie dominante ${ }^{32}$ »Cela se traduit donc par une fermeture du débat, un déni de l'expérience et de la parole. " L'expression débridée de la souffrance a tendance à se clore sur elle même et rend difficile le discours sur les causes et le nécessité d'une riposte collective " ${ }^{33}$.

31 De mode de co-existence, la rationalité instrumentale devient un mécanisme de défense pour se protéger du conflit et de la confrontation avec l'autre. Elle se révèle être un déni de l'altérité. C'est l'omnipotence de la pensée orientée vers la seule recherche de l'efficacité.

32 Ne pas accepter le paradoxe au fondement de la coexistence organisationnelle en survalorisant la logique instrumentale, c'est générer une violence qui en lin de compte vient détruire toute l'orme de reconnaissance de l'autre. C'est une des formes des plus subtiles du déni de la mort, de la séparation et de la violence. Cette défense générant la mort de l'antre et la violence organisationnelle ${ }^{34}$.

\section{La coexistence instrumentale : une fusion dangereuse}

L'axe de l'altérité/identité est l'impensé puisque l'autre comme altérité est nié, or c'est la rencontre de l'altérité qui m'engage dans la responsabilitée ${ }^{35}$. La dynamique psychique qui prévaut est celle de l'omnipotence et du narcissisme: l'autre est alors un objet mis en discours, rapporté, évalué (comme dans une grille d'employabilité). L'espace fusionnel qui en découle est un espace régressif où se confond la régression infantile avec une figure maternante "institutionnalisée ». La substitution d'une emprise molle à une emprise incarnée par une relation autoritaire correspond à la croyance "que tous les conflits peuvent se résoudre par une assistante maternelle publique qui absout l'individu de toute responsabilité morale et le traite comme une victime des conditions sociales ${ }^{36} »$. Cette fusion dangereuse empêche la décision de reconnaître l'autre et de laisser l'attachement à une mère perdue à jamais. Dans cette fusion, le tiers séparateur (silence des pairs lors de harcèlement) est absent. "Le collectif se délite et les rapports sociaux dégénèrent en rapports 
interindividuels où le face-à-face ne parvient plus à se distancier et à se réguler en référence à une instance tierce $\|^{37}$

\section{BIBLIOGRAPHIE}

ARDOINO J., Le sujet aux regards doubles de la clinique psychosociologique et de la recherche psychosociale, in L 'aventure Psychosociologique (dir.) Aubert N.. et alii. Desclée de Brouwer. 1997. BARUS MICHEL J., Intervenir face aux paradoxes de l'organisation et aux dérobades de l'idéal. Journées IPM. Toulouse. 1997.

BARUS MICHEL J.. Sens ou efficience. Démarche clinique et rationalité instrumentale, in Revue Internationale de Psychosociologie. 1997. vol. III. n S. ESKA.

BELLENGER L... Etre pro. ESF. 1992.

BERGMANN A.. Ethique et gestion, dans Encyclopédie de gestion. Economica. 1989.

BERGSON H., Les deux sources de la morale et de la religion. PUF. 1984.

BOLTANSKI L., CHIAPELLO C. Le nouvel esprit du capitalisme. Gallimard, nrf. 1999.

CASTORIADIS C.. La rationalité du capitalisme, in Revue Internationale de

Psychosociologie. 1997. vol. III. n 8. éd. ESKA.

DEJOURS C. Souffrance en France. Seuil. 1998.

DUBOULOY M.. Deuil et coaching. Journées IPM. Toulouse. 1997.

DUBOULOY M.. LABRE C. Les restructurations d'entreprise : de la rationalité économique à la souffrance des hommes. Journées IPM. HEC.

Jouy-en-Josas. Juin 2000.

GALAMBAUD B., Une nouvelle configuration humaine de l'entreprise. ESF. 1994.

GUIST-DESPRAIRIES F.. La rationalité comme défense dans la relation éducative. Revue Internationale de Psychosociologie. 1997. vol. III. nº 8. éd. ESKA.

IBANEZ T. faire et croire. Perspectives cognitives et conduites sociales. numéro 3. DelVal. 1989.

LE GOFF, Revue Débat numéro 123. janvier février 2003.

SAIELLI P.. Lu rationalité instrumentale comme défense contre l'angoisse.

Congrès IPM. Pau 2003.

SAIELL\$I P., De la normalisation de la démarche éthique. Revue Communication ci Organisation. GREC/O. ISIC. Université Michel de Montaigne Bordeaux.3. $n^{\circ} 17.2^{\mathrm{e}}$ Semestre 2000

SCHNEIDER M. ., Big Mother (Psychopathologie de la vie politique), éd. Odile Jacob. 2002.

VIGNON C. SCOYEZ S., GILLET I.. Représentations de l'autonomie et autonomie en représentation, colloque IEEE. 2002. 


\section{NOTES}

1. La patemité du concept « rationalité instrumentale» revient à l'école de Francfort (Adorno. Habermas). Elle interprète ainsi la rationalisation wébérienne en soulignant la toute puissance de la logique capitaliste qui diffuse une logique moyens-fins dans une volonté de maîtrise du temps en rupture avec une rationalité axiologique.

2. Morgan K. Images de l'organisation. Editions. ESKA. 1989

3. La resistitble emprise de la rationalité instrumentale, (dir) Guist-Desprairies F., Lévy A.. NicolaïA. Revue Internationale de Psychosociologie, 1997, vol. III. $n^{\circ}$ 8. éd. ESK.A. p. 7.

4. Barus Michel.J.. Sens ou efficience Démarche clinique et rationalité instrumentale, dans Revue Internationale de Psychosociologie. 1997. vol. III. $\mathrm{n}^{\circ}$ 8, éd. ESKA

5. Barus Michel.1. Intervenir face aux paradoxes de l'organisation et aux dérobades de l'ideal. Journées IPM. Toulouse. 1997

6. Guist-Desprairies F., La rationalité comme défense dans la relation éducative. Revue Internationale de Psychosociologie, 1997. vol. III. $\mathrm{n}^{\circ}$ 8. éd. ESKA

7. Barus Michel J.,op.cit.

8. Castoriadis C. La rationalité du capitalisme, dans Revue Internationale de Psychosociologie, 1997. vol. III. $n^{\circ}$ 8. éd. I SKA.

9. Castoriadis $C$, op. cit. p. 37

10. Barus-Michel J. op. cit. p. 65

11. Guist-Desprairies F., op. cit.

12. Guist-Despraines F. op. cit p. 90

13. Saielli P..La rationalité comme défense contre l'angoisse. Congrès IPM. Pau 2003

14. Arduino.J. Le sujet aux regards doubles de la clinique psychosociologique et de la recherche psychosociale, dans L'aventure Psychosociologique (diri Aubert N.. et alii. éd Desclée de Brouwer. 1997. pp. 125-131

15. Duboulov M. et labre C. les restructurations d'entreprise de la rationalité économique a la souffrance des hommes Journées IPM HEC Jouy-en-Josas. Juin 2000. p. 7

16. Cf. Dejours C (1998). Souffrance en France, éd du Seuil. Paris et Duboulov M (1997). Deuil et coaching. in Actes du colloque de l'IPM. 1997

17. Ariès P., Harcèlement au travail ou nouveau management. Editions Golias, 2002. p. 270.

18. Nous empruntons l'expression à.JP Boutinet. Questionnement anthropologique autour de l'accompagnement. Education permanente $\mathrm{n}^{\circ}$ 153. page 246. Le projet, une «figure idéalisée » dans le cadre de l'aide sociale et dans les dispositifs d'accompagnement des «vulnérables ». a connu un repli dans ses ambitions dans les années 2000 : l'accompagné se retrouve passif et «dans l'obligation de se doter d'un projet au regard d'un nouvel imperatif de mobilité » dans une société postmoderne oscillant entre deux symptômes : dépression et cynisme

19. Vignon. Scoyez Gillet. Représentations de l'autonomie et autonomie en représentation. Colloque IEEE. 2002. Les raisonnements d'un interviewé prend ainsi des allures de tautologie : « il est autonome parce qu'il est rapide, il est rapide parce qu'il est autonome ».

20. Ibanez T. faire et croire. Perspectives cognitives et conduites sociales, numéro 3. Del Val. 1989

21. Ardoino.J.Le sujet aux regards doubles de la clinique psychosociologique et de la recherche psychosociale, dans L'aventure Psychosociologique ( $\mathrm{dm}$ Aubert N.. et alii, éd Desclée de Brouwer. 1997. p. 129

22. Boltanski Chiapello. Le nouvel esprit du capitalisme, nrf 1999

23. Boltanski Chiapello. op cité p. 187.

24. Boltanski Chiapello. op cité, p. 157

25. Bellenger 1., Etre pro. ESF, 1992. 
26. Boltanski Chiapello, op. cit. p 164

27. Boltanski Chiapello., op. cit. p. 186 et 187.

28. Bergmann A. Éthique et gestion, dans Encyclopédie de gestion, éd. Económica. 1480. p. 1118.

29. Saielli P., De la normalisation de la démarche éthique. Revue Communication et Organisation, $\mathrm{n}^{\circ}$ 17. $2^{\mathrm{e}}$ Semestre 2000

30. Bergson H.. Les deux sources de la morale et de la religion, éd. PUF, 1984

31. Galambaud B.. Une nouvelle configuration humaine de l'entreprise, éd. ESF, 1994.

32. Schneider M. Big Mother (Psychopathologie de la vie politique), éd. Odile Jacob. 2002. p. 72

33. Le Goff. Revue Débat numéro 123. Janvier/février 2003

34. Article du Monde du 11/02/03.

35. Lévinas E...Humanisme de l'autre homme, éd, Fata Morgana. 1472

36. Schneider M. Big Mother (Psychopathologie de la vie politique), éd. Odile Jacob.

2002 p. 163.

37. Le Goff. Post lace 2000 a l'édition Les illusions du management, la découverte première édition 1997. p. 158

\section{AUTEURS}

PHILIPPE SAIELLI

Université de Valenciennes, LARIME

\section{ISABELLE GILLET-ROUSSEL}

Université Lille 2, GERICO 